\title{
Morfología e inmunofenotipo de las células plasmáticas en el mieloma múltiple
}

\author{
Morphology and immunophenotype \\ of plasma cells in multiple myeloma
}

\section{Nataly J. Rincón-Vásquez MB', Patricia E. Jaramillo-Arbeláez Msc², Carlos M. Llanos-Albornoz TM³}

Resumen: el avance en el conocimiento de la fisiopatología del mieloma múltiple ha permitido el acercamiento a la comprensión de su heterogeneidad, en términos del comportamiento clínico, citomorfológico, fenotípico, bioquímico, genético y molecular, lo que contribuye al desarrollo de nuevos tratamientos y al establecimiento de modelos que apuntan hacia una cronicidad de la enfermedad. Los cambios morfológicos de la célula plasmática en el mieloma múltiple indican, principalmente, alteraciones a nivel del núcleo, que orientan a la diferenciación de las células reactivas de aquellas clonales; además, que su inmadurez es de mal pronóstico. Igualmente, la expresión de ciertos marcadores monoclonales, y la ausencia de otros, detectados mediante citometría de flujo, clasifican inmunofenotipicamente a las células neoplásicas del mieloma múltiple, lo que permite considerar esta herramienta como una de las mejores en el diagnóstico y en el manejo de la enfermedad mínima residual. En esta revisión se pretende enfatizar en la importancia de identificar los cambios morfológicos que se dan en las células plasmáticas y su relación con las características inmunofenotípicas en las neoplasias, que tienen una asociación pronóstica importante en el desarrollo del mieloma múltiple.

Palabras clave: mieloma múltiple, inmunofenotipo, morfología, células plasmáticas, citometría de flujo, médula ósea.

Rincón-Vásquez NJ, Jaramillo-Arbeláez PE, Llanos-Albornoz CM. Morfología e inmunofenotipo de las células plasmáticas en el mieloma múltiple. Medicina \& Laboratorio 2017; 23: 443-458.

${ }^{1}$ Microbióloga y Bioanalista, estudiante de maestría en Microbiología y Bioanálisis, con énfasis en Hematología,

Universidad de Antioquia. Bacterióloga, área de Hematología, Laboratorio Clínico, Hospital General de Medellín. Medellin, Colombia.

${ }^{2}$ Bacterióloga, especialista en Hematología, MSc en Microbiología con énfasis en Hematología y en Bioética. Docente de Hematología, Universidad de Antioquia. Medellín, Colombia. Correo electrónico: patelen17@gmail.com

${ }^{3}$ Tecnólogo Médico. Director del Centro de Investigación, Desarrollo e Innovación INCA. Lima, Perú. 
$\mathbf{E}$ mieloma múltiple es una neoplasia hematológica que cursa con una proliferación clonal de las células plasmáticas malignas, que se acumulan en la médula ósea y producen altas cantidades de una paraproteína o inmunoglobulina monoclonal, la proteína $\mathrm{M}$, lo que da lugar a citopenias y resorción ósea [1-3]. Esta alteración hematológica fue clasificada, por la Organización Mundial de la Salud, en el 2016, dentro del grupo de «neoplasias de células B maduras», como mieloma de células plasmáticas [4]. Esta entidad está acompañada de una serie compleja de manifestaciones clínicas conocidas bajo el acrónimo en inglés CRAB: calcio elevado en suero, insuficiencia renal, anemia, lesiones líticas de hueso u osteoporosis severa $[5,6]$, y ha sido relacionada con la coexistencia o aparición de otras neoplasias hematológicas, como los síndromes mielodisplásicos, la leucemia mieloide aguda [7-9] y la leucemia neutrofílica crónica [10].

Según su distribución mundial, el mieloma múltiple tiene una incidencia anual estandarizada de 1,5 casos por 100.000 individuos, y una prevalencia a cinco años de aproximadamente 230.000 pacientes [11]. Este afecta, principalmente, a la población con una edad promedio de 65 años y es ligeramente más frecuente en el sexo masculino y personas de raza negra [12]. En pacientes menores de 30 años es extremadamente raro $[13,14]$, al igual que en mujeres embarazadas [15]. El mieloma múltiple representa el $1 \%$ de todas las neoplasias y entre el $10 \%$ y el $12 \%$ de las neoplasias hematológicas; la segunda más frecuente después del linfoma no Hodgkin [16-18]. Para 2016 se estimaron entre 24.280 y 30.330 nuevos casos, y 12.650 muertes [19,20]. El riesgo de desarrollar mieloma múltiple se cree que es 3,7 veces mayor para los individuos con un familiar de primer grado de consanguinidad que haya padecido la enfermedad [21].

Según el proyecto GLOBOCAN 2012, de la Agencia Internacional para la Investigación en Cáncer (IARC, del inglés, International Agency for Research on Cancer), la incidencia anual estandarizada del mieloma múltiple en Colombia fue de 1,4 casos por 100.000 individuos, lo que lo ubica como el tercer país de Sudamérica con mayor cantidad de casos registrados, luego de Brasil y Argentina [22]. Por su parte, de acuerdo con la proyección de crecimiento demográfico en Colombia de este proyecto, para el 2015 se presentarían alrededor de 681 nuevos casos y 530 muertes de personas afectadas por mieloma múltiple, y para 2020 ascenderían a 815 nuevos casos y 647 muertes [23].

En los últimos años, las nuevas tecnologías han logrado que alrededor del $30 \%$ de los pacientes diagnosticados con mieloma múltiple alcancen una tasa de supervivencia global cercana a los 10 años, tres veces más que lo reportado a inicios del 2000 [24-27]. No obstante, esta es una enfermedad incurable que genera una serie de incapacidades en los pacientes que la padecen, aun encontrándose en edad productiva, lo que, sumado a los altos costos y la baja disponibilidad de los tratamientos, genera un impacto importante sobre la economía y el sistema de salud de los países en vía de desarrollo [28,29].

Por lo tanto, la identificación de nuevos marcadores pronósticos y una intervención temprana tendrá impacto esencial sobre la calidad de vida de los pacientes y sobre los recursos económicos que son destinados a la salud en Colombia. En esta revisión se presenta información sobre el proceso de producción y maduración de las células plasmáticas, así como las alteraciones mor- 
fológicas más notorias y las características inmunofenotípicas relacionadas con su proliferación y maduración aberrante en el mieloma múltiple.

\section{Maduración normal de las células plasmáticas}

Las células plasmáticas son el producto final de la diferenciación del linfocito $B$, evento íntimamente relacionado con la expresión ectópica del represor transcripcional BLIMP1 y XBP1 [30]. Su principal función es la producción de anticuerpos, para lo cual requiere de varias etapas. Inicialmente, este es un proceso independiente de la exposición al antígeno, que abarca el desarrollo temprano y la determinación de la diferenciación de la célula madre pluripotencial en médula ósea hacia células del linaje $B$, que depende de la participación clave de los factores de transcripción PU.1, E2A, EBF y PAX5 [31]. Seguido, se encuentra el reordenamiento de los segmentos del gen de la cadena pesada de la inmunoglobulina (IgH) en las células pro- $B$, que permite su paso a las células precursoras pre- $B$, las cuales expresan $\mu \mathrm{M}$ (forma transmembrana de la cadena pesada de la inmunoglobulina $\mu$ ), que proporciona, en el receptor de células pre-B (pre-BCR), el primer punto de control para el desarrollo de las células $B$, que lleva a la expansión clonal y el reordenamiento de los segmentos del gen de la cadena ligera de la inmunoglobulina (IgL) [32].

Para pasar al segundo punto de control se requiere la presencia de la inmunoglobulina $M$ (IgM) de superficie, que permite que los linfocitos $\mathrm{B}$ vírgenes $\operatorname{lgM}^{+}$sobrevivan a la selección negativa, salgan de la médula ósea hacia el bazo y los nódulos linfáticos, donde se va a dar la exposición antigénica que estimula su diferenciación hacia la célula plasmática [32]. Para ello, los linfocitos B reconocen al antígeno en forma soluble por medio de las inmunoglobulinas de membrana, que forman parte del receptor de las células $B$, conocido como BCR. En cada linfocito hay entre 50.000 y 100.000 moléculas de inmunoglobulinas de membrana (de las clases $M$ y D), que han sido sintetizadas por él mismo; todas ellas con igual especificidad antigénica. En ausencia de estímulo antigénico, los linfocitos $B$ maduros vírgenes mueren por apoptosis al cabo de unos pocos días. Sin embargo, otros se unen por su BCR al antígeno complementario específico y, con la ayuda de señales de los macrófagos y las células $T$, inician la selección y proliferación clonal, que termina (al cabo de cuatro a cinco días) con la diferenciación hacia dos subpoblaciones, una de células plasmáticas secretoras de anticuerpos y otra de células B de memoria (véase figura 1) [33].

\section{Características morfofuncionales de las células plasmáticas}

La transformación de los linfocitos $B$ a células plasmáticas trae como consecuencia un evidente cambio en sus características morfofuncionales, entre las que se encuentran [34]:

- Carecen de inmunoglobulina de membrana.

Son de mayor tamaño y con mayor proporción de citoplasma que las células $B$ de las que proceden.

Su retículo endoplasmático rugoso está muy desarrollado, así como su aparato de Golgi, lo que explica la gran cantidad de anticuerpos que producen y son secretados, los cuales poseen la misma especificidad antigénica que la de las inmunoglobulinas de membrana de la célula B original. 


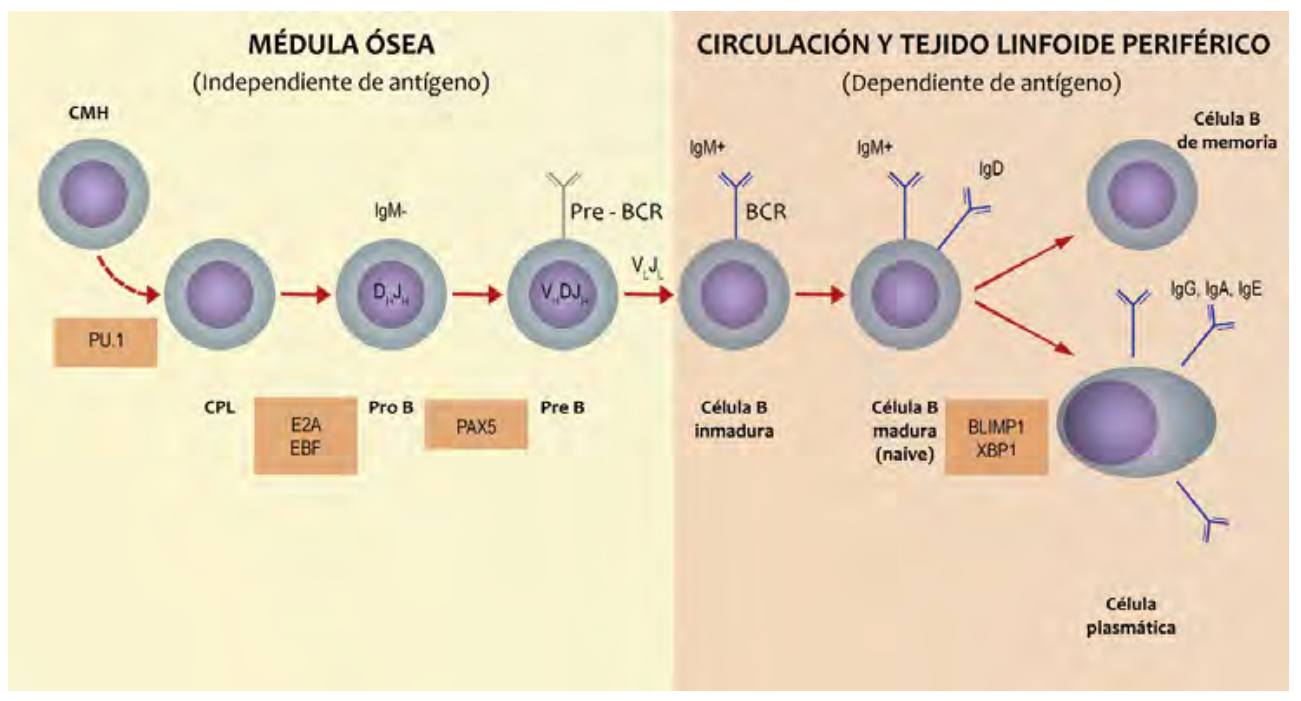

Figura 1. Ciclo madurativo de las células plasmáticas. $\mathrm{CMH}=$ célula madre hematopoyética, $\mathrm{CPL}=\mathrm{célula}$ progenitora linfoide.

- No circulan por la sangre ni por los vasos linfáticos, sino que se localizan en los órganos linfoides secundarios y los lugares de la respuesta inmunológica.

- Viven unos pocos días, pues al ser células en una fase de diferenciación terminal carecen de capacidad mitótica y mueren por apoptosis; mientras que los linfocitos $B$ de memoria pueden mantenerse en estado de reposo (G0) por largos periodos de tiempo (más de 20 o 30 años).

- Cuando se exponen al antígeno específico dan una respuesta inmunitaria más rápida, más intensa y con mayor afinidad.

Su aspecto es similar al de los linfocitos B vírgenes.

\section{Morfología de las células plasmáticas}

Las células plasmáticas maduras constituyen, normalmente, menos del $1 \%$ de las células en la médula ósea. Su morfología es variada en los frotis coloreados con derivados de Romanowsky, y su tamaño varía entre $15 \mu \mathrm{m}$ y $30 \mu \mathrm{m}$ de diámetro. Estas células poseen un citoplasma abundante, muy basófilo y azul profundo, lo que se debe al retículo endoplasmático rugoso de gran extensión que poseen, el cual, a su vez, les da la capacidad para sintetizar gran cantidad de proteínas. Además, presentan una zona perinuclear incolora, que corresponde al lugar que ocupa el aparato de Golgi, el cual puede tener excepcionalmente una pequeña vacuola lipídica denominada cuerpo de Gall, y tienen un núcleo redondo u oval, con cromatina densamente condensada, excéntrico y pequeño en relación con el citoplasma [35]. 


\section{Estadios de maduración}

La maduración normal de la línea plasmática se compone de tres estadios [36]:

1. Plasmoblasto: presentan un tamaño de $15 \mu \mathrm{m}$ a $25 \mu \mathrm{m}$, un núcleo redondo a oval, excéntrico, con cromatina reticulada y paracromatina bien delimitada, dos a cuatro nucléolos, citoplasma entre moderado e intensamente basófilo y sin gránulos.

2. Proplasmocito: presentan un tamaño de $15 \mu \mathrm{m}$ a $25 \mu \mathrm{m}$, un núcleo ovalado a redondo, excéntrico y con cromatina moderadamente burda, uno a dos nucléolos y citoplasma azul con zona perinuclear más clara. La típica basofilia brillante del plasmocito suele aparecer desde este precursor, por lo que tiende a parecerse a un normoblasto basófilo, pero, generalmente, la tonalidad azul de este último es más oscura.

3. Plasmocito: tiene un tamaño de $10 \mu \mathrm{m}$ a $20 \mu \mathrm{m}$, un núcleo redondo u oval, excéntrico, con cromatina burda y en acúmulo y paracromatina definida, pero dispersa, sin nucléolo evidente y un citoplasma azul con una zona perinuclear más clara.

Ocasionalmente, una pequeña proporción de células plasmáticas normales puede mostrar varias características morfológicas, producto de los estímulos antigénicos que sufre al salir de la médula ósea, como son los cuerpos de Russell, los cuales corresponden a grandes inclusiones citoplasmáticas homogéneas eosinofílicas formadas por la acumulación intracelular de las inmunoglobulinas; en este caso, las células plasmáticas reciben el nombre de células de Mott o morulares. Dichas inclusiones de inmunoglobulinas también se pueden observar, ocasionalmente, como estructuras cristalinas en el citoplasma. Además, las células plasmáticas pueden observarse con un citoplasma rojo-rosado con la tinción de May Grünwald-Giemsa; caso en el cual se conocen como "células flameadas» [37].

\section{Células plasmáticas en el mieloma múltiple}

Las células plasmáticas en el mieloma múltiple alteran la homeostasis de las células estromales y su interacción con la matriz extracelular, las citoquinas y los factores de crecimiento dentro del microambiente hematopoyético, lo que en consecuencia desencadena una señalización molecular anómala que promueve la proliferación, la supervivencia y la migración celular, al igual que la alteración de los mecanismos de autofagia, sistema ubiquitina-proteosoma y respuesta a proteínas mal plegadas, lo que influye en la resistencia a los medicamentos y la progresión de la enfermedad [38-40].

Generalmente, las anomalías morfológicas de las células plasmáticas se pueden dividir en:

Alteraciones con cambios en el citoplasma, como la coloración acidófila y la presencia de inclusiones formadas a causa del metabolismo anormal de las inmunoglobulinas sintetizadas por las células plasmáticas, que se observan tanto en los trastornos reactivos como neoplásicos.

Alteraciones relacionadas con el núcleo, que corresponden a una condensación anormal de la cromatina, un nucléolo prominente y un contorno nuclear irregular, que son propias de las células malignas [41] (véase figura 2). 
A

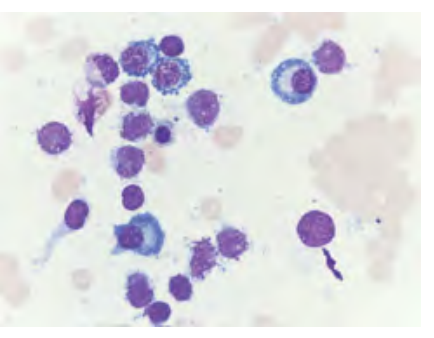

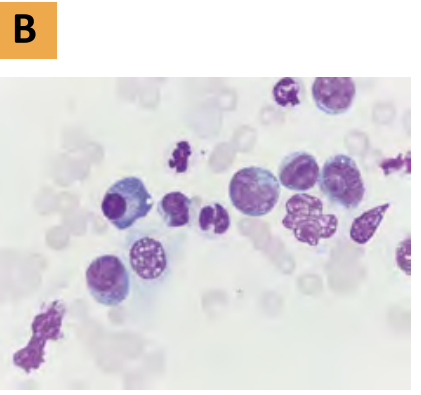

C

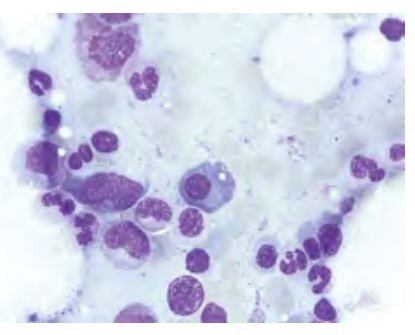

Figura 2. Plasmocitos con coloración de Wright en muestra de aspirado de médula ósea, 100X. A. Plasmocitos maduros con espacio citoplasmático que corresponde al aparto de Golgi. B. Proplasmocitos con presencia de nucléolos, abundante citoplasma y cromatina con maduración intermedia. C. Plasmocitos maduros con mayor tamaño y fragmentación de cromatina, vacuola citoplasmática y bordes irregulares. Cortesía de la Universidad de Antioquia, Medellín, Colombia.

\section{Alteraciones morfológicas del citoplasma de la célula plasmática}

Se han podido evidenciar algunas características atípicas propias de la malignidad de las células plasmáticas, tales como los cuerpos de Dutcher, los cuerpos de Russell y los bastones azurófilos, que tienen ultraestructura cristalina similar a los cuerpos de Auer [42-44], las cuales han mostrado positividad para la reacción con peroxidasa [45]. A continuación, se describen algunas de estas alteraciones citoplasmáticas en las células plasmáticas:

Cuerpos de Dutcher: son acumulaciones intracitoplasmáticas de inmunoglobulinas que aparecen como inclusiones invaginadas hacia o sobre el núcleo. Estos se presentan únicos o no, son de color gris claro, su tamaño es variable, incluso pueden alcanzar a ocupar la totalidad del núcleo y aparentar ser, en ocasiones, un nucléolo grande. En muestras de biopsia de médula ósea se tiñen de color rosa con la tinción de hematoxilina y eosina, y muestran una coloración variable con el ácido peryódico de Schiff
(PAS). Se observan frecuente, pero no exclusivamente, en el mieloma múltiple [46].

Cuerpos de Russell: son acumulaciones intracitoplasmáticas de las inmunoglobulinas sintetizadas por la célula plasmática, que se observan como esférulas citoplasmáticas grandes que a menudo desplazan al núcleo. Por lo regular son únicas por célula, pueden medir hasta $20 \mu \mathrm{m}$ de diámetro, tienen una coloración de rojo oscuro a amarillo hialino, la coloración con ácido peryódico de Schiff (PAS) puede ser positiva a variable. Estos cuerpos resultan de la condensación de la inmunoglobulina dentro de las cisternas distendidas del retículo endoplásmico rugoso y se pueden observar tanto en las gammapatías malignas como reactivas $[41,47]$.

Células de Mott o células morulares: corresponden a las células plasmáticas que contienen un número variable (hasta 100) de cuerpos de Russell en su citoplasma y presentan un tamaño de $1 \mu \mathrm{m}$ a $5 \mu \mathrm{m}$ de diámetro; son ligeramente basofílicas, pero a veces pueden ser incoloras, grises, rosadas o eosinofílicas. Este tipo de células 
se pueden observar en procesos reactivos, principalmente infecciosos o inflamatorios, así como en la gammapatía monoclonal de significado incierto (GMSI) y en el mieloma múltiple [41].

Células flameadas o tesaurocitos: se nombran así a las células plasmáticas que adquieren un color rojizo o magenta en su citoplasma, con un tono carmín en la periferia, al ser coloreados con la tinción de May Grünwald-Giemsa, pero que pueden adquirir un tono eosinofílico al ser teñido con hematoxilina y eosina. Este color de su citoplasma está relacionado con la dilatación de las cisternas del retículo endoplasmático rugoso, debido a la acumulación de inmunoglobulinas con alto contenido de carbohidratos. Esta alteración ha sido asociada frecuentemente al mieloma múltiple tipo IgA, pero se puede observar en las gammapatías de otras clases de inmunoglobulinas, e incluso en condiciones reactivas $[41,48]$.

Inclusiones cristalinas: son varillas azurófilas con una ultraestructura cristalina, algunas similares a bastones de Auer, que suelen ser alargadas y delgadas, y que están ubicadas de manera dispersa en el citoplasma de las células plasmáticas o agrupadas en haces. El color de estas inclusiones puede variar desde rojo púrpura (azurofílico), rosado, azul morado hasta incluso incoloro. Las inclusiones similares a los cuerpos de Auer son hallazgos morfológicos muy raros y su etiología aún es confusa, aunque en algunos casos se ha considerado que pueden ser de procedencia lisosomal. Estas inclusiones son generalmente negativas bajo las coloraciones de ácido peryódico de Schiff (PAS), sudán negro y mieloperoxidasa $[41,42]$ (véase figura $\mathbf{3 G}$ y $\mathbf{3 H}$ ).

\section{Alteraciones morfológicas del núcleo de la célula plasmática}

Entre las alteraciones morfológicas del núcleo de las células plasmáticas en el mieloma múltiple se han descrito:

Células plasmáticas con contorno nuclear irregular: son células plasmáticas con núcleos clivados, con muescas o «blebs», lo cual se ha relacionado con hipodiploidía, un cambio citogenético asociado con pronóstico adverso en el mieloma múltiple [41]. Los análisis morfométricos de células plasmáticas en el mieloma múltiple han demostrado que estas células pueden ser indicativas de un estadio más avanzado de la enfermedad, que se asocia también a peor pronóstico [49].

Células plasmáticas bi o multinucleadas: las células plasmáticas binucleadas cuentan con dos núcleos, ambos con un tamaño y patrón de cromatina similares. Se habla de malignidad si estas células plasmáticas presentan al menos uno de los siguientes criterios: tres núcleos o más, núcleos que difieren en tamaño, presencia de nucléolo o una cromatina finamente dispersa. Sin embargo, en la mayoría de los casos, las células plasmáticas multinucleadas corresponden solo a una pequeña parte del porcentaje observado de células en médula ósea; además, se puede pensar en un diagnóstico de asincronía de células plasmáticas, sin estar asociado a un mieloma múltiple, ya que han sido reportadas en médulas óseas normales, en desórdenes reactivos y en la gammapatía monoclonal de significado incierto $[50,51]$ (véase figura 4).

En las décadas anteriores, a través de diversos estudios, se ha informado que la morfología de las células plasmáticas ayu- 


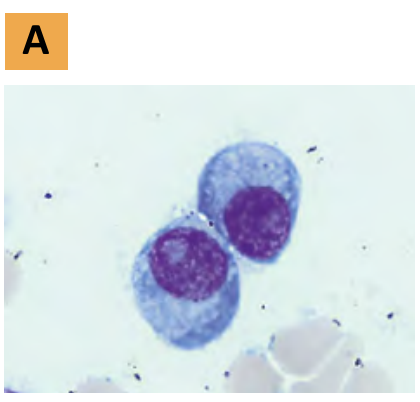

D

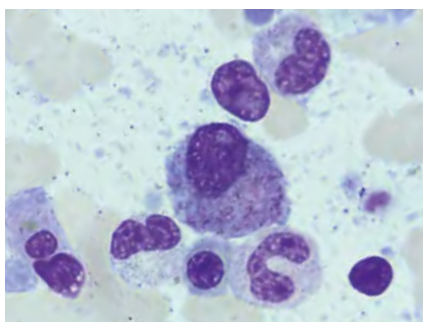

G

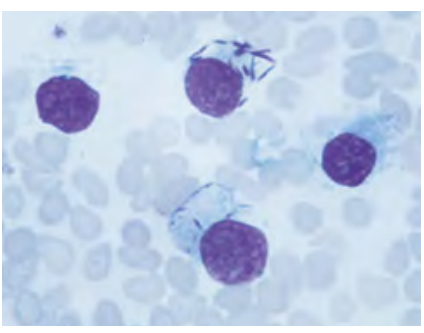

B

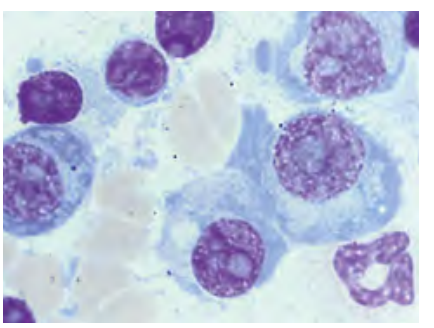

E

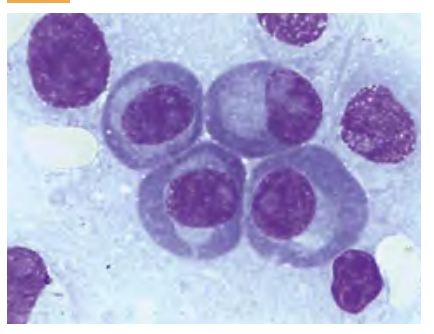

H

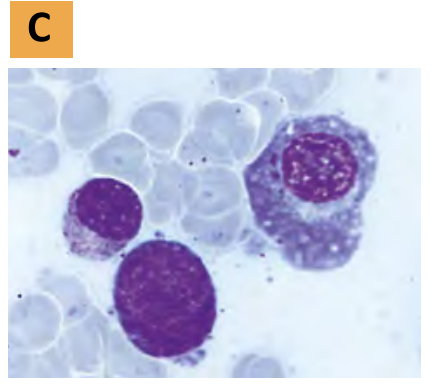

F

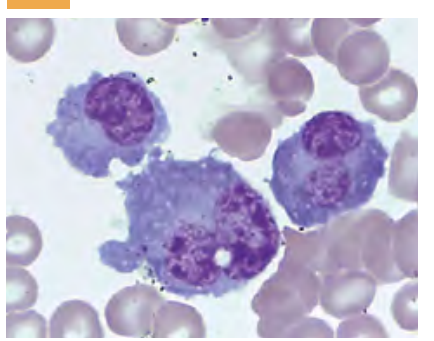

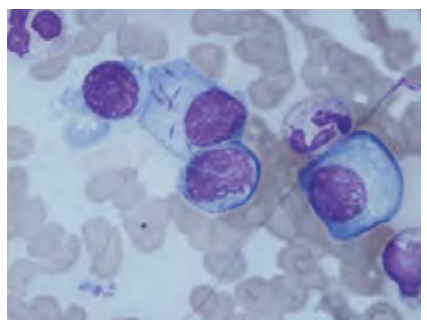

Figura 3. Plasmocitos con coloración de Wright en muestra de aspirado de médula ósea con alteraciones citoplasmáticas, 100X. A-B. Obsérvese citoplasma abundante, pérdida del espacio del aparato de Golgi y borde citoplasmático irregular. C-D. Obsérvense gránulos y vacuolas lipídicas, E. Obsérvese basofilia citoplasmática, F. Obsérvense bordes citoplasmáticos y nucleares irregulares. G-H. Obsérvense agujas o astillas de inmunoglobulinas similares a cuerpos de Auer. Cortesía de la Universidad de Antioquia, Medellín, Colombia.

da a predecir el pronóstico del paciente con mieloma múltiple [52-55]; incluso, se desarrolló una clasificación morfológica de las células plasmáticas encontradas en esta enfermedad y se determinó que la presencia del subtipo plasmoblástico está relacionado con tasas de supervivencia significativamente más cortas, comparado con el hallazgo de solo células plasmáticas maduras [56] (véase figura 5). Por lo tanto, la correcta descripción de los cambios morfológicos identificados durante la visualización de estas células conduce a la realización de un recuento en frotis de aspirados de médula ósea confiable, donde la determinación de las alteraciones citoplasmáticas y nucleares van a ser cruciales para el diagnóstico de mieloma múltiple. 

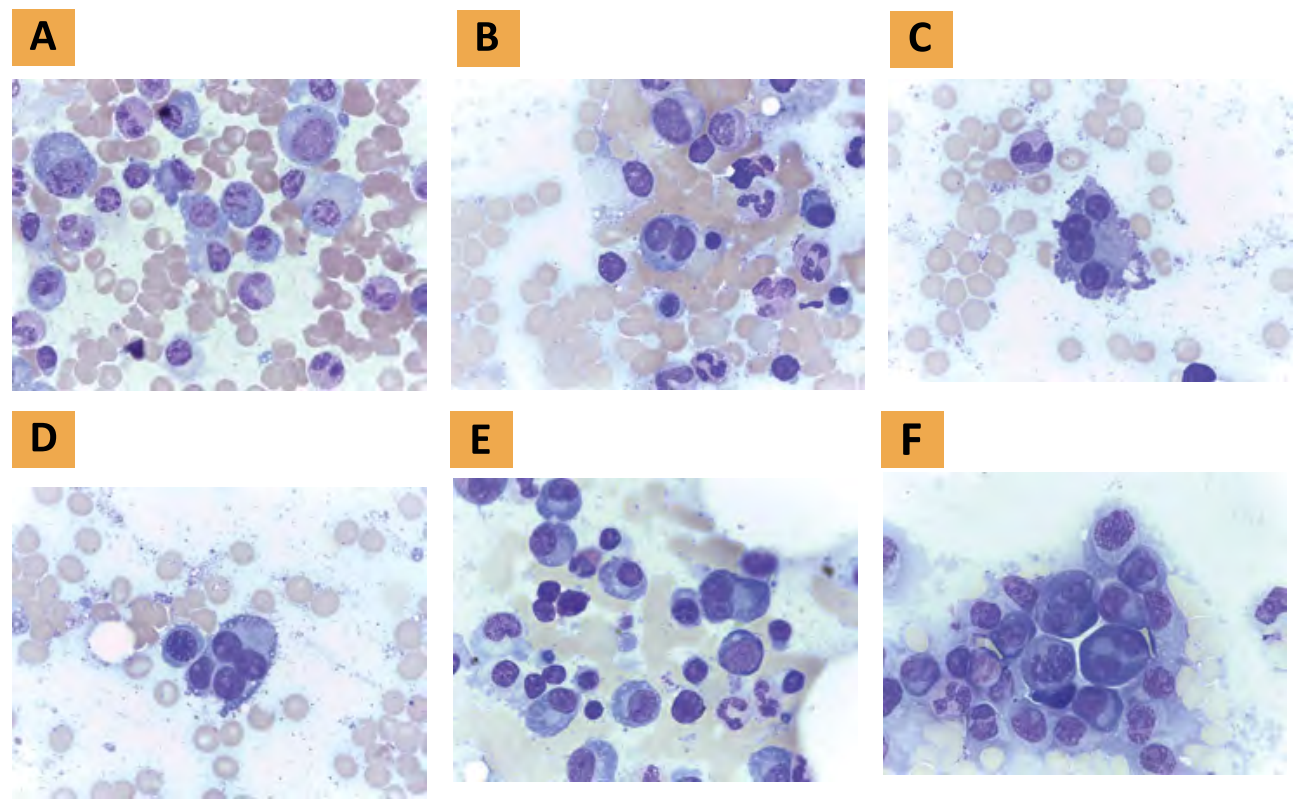

Figura 4. Plasmocitos con coloración de Wright en muestra de aspirado de médula ósea con alteraciones nucleares, 100X. A. Obsérvese cromatina irregular. B-D. Obsérvese binucleación/multinucleación. E-F. Obsérvense formas aberrantes del núcleo. Cortesía de la Universidad de Antioquia, Medellín, Colombia.

\section{Inmunofenotipo de las células plasmáticas}

La determinación del inmunofenotipo por citometría de flujo es útil para caracterizar fenotípicamente y distinguir las células plasmáticas patológicas de las sanas o reactivas. Además, proporciona información de marcadores celulares con utilidad para el pronóstico clínico [57]. En algunas ocasiones se observa diferencia entre el recuento celular por aspirado de médula ósea y el realizado por citometría de flujo, debido, probablemente, a la fragilidad que tienen las células plasmáticas, que al ser sometidas al proceso preanalítico de la citometría de flujo disminuyen su cantidad, y a las diferencias propias de las muestras recolectadas durante el aspirado.
Las células plasmáticas se caracterizan, esencialmente, por la coexpresión de las moléculas de membrana CD138 (sindecano-1) y $\mathrm{CD} 38$, lo que permite su identificación mediante citometría de flujo en muestras provenientes de médula ósea, sangre periférica o suspensiones celulares de tejidos. Las células plasmáticas pueden perder la expresión de algunos marcadores empleados para evaluar la población linfoide B (pan-B), como las moléculas CD19, CD20 y CD22, y las inmunoglobulinas de membrana, pero conservar la expresión de la molécula CD27 [58].

La Red Europea de Mieloma (EMN, del inglés, European Myeloma Network) recomienda estudiar por citometría de flujo los antígenos CD19/CD56/CD117/CD20/CD28/ CD27/CD81/CD200 para el diagnóstico del mieloma múltiple, la gammapatía monoclonal de significado incierto y las condicio- 


\section{A}

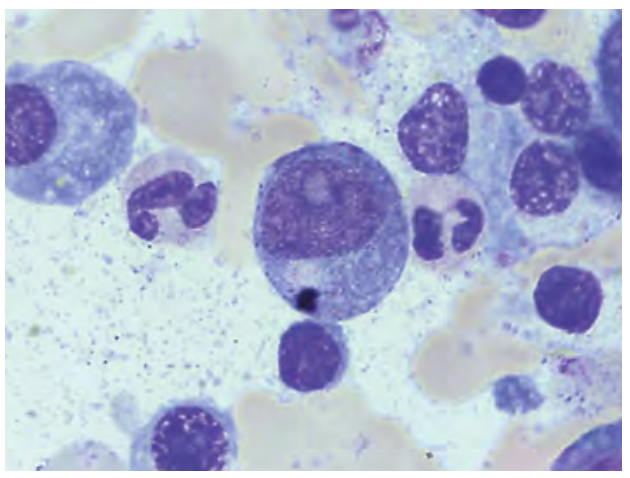

C

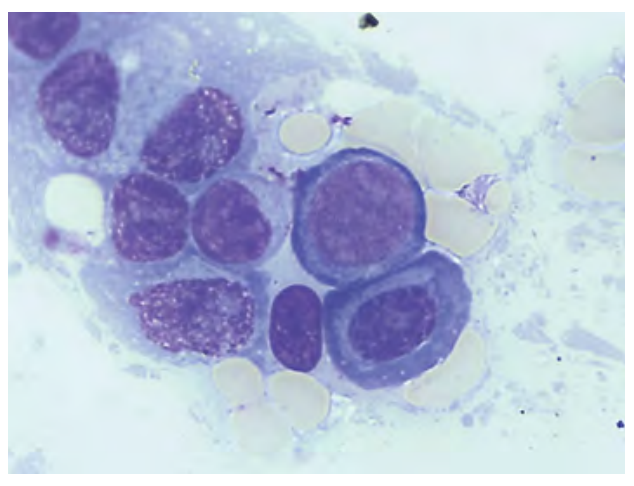

\section{B}

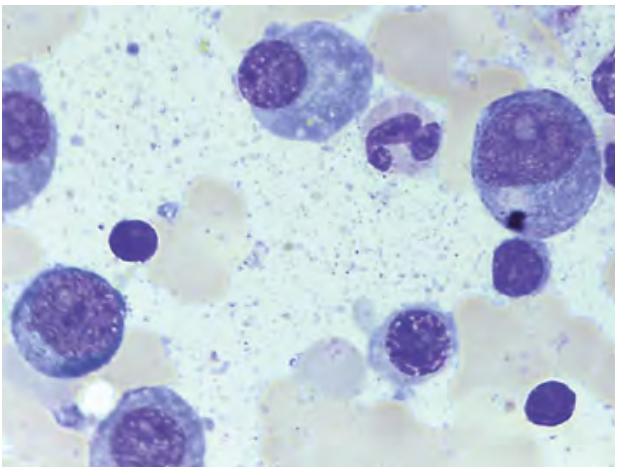

D

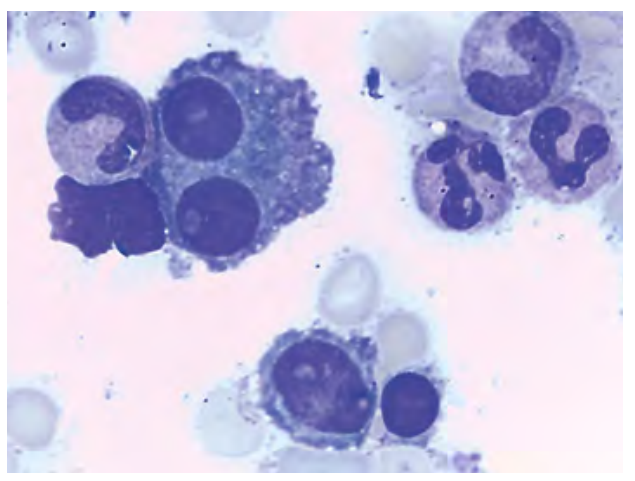

Figura 5. Coloración de Wright en muestras de aspirado de médula ósea de diferentes casos con diagnóstico de mieloma múltiple, 100X. Obsérvese presencia de células plasmoblásticas con nucléolos evidentes y relación núcleo/citoplasma aumentada. Cortesía de la Universidad de Antioquia, Medellín, Colombia.

nes reactivas, y los antígenos CD45/CD56/ CD117/CD28 como marcadores pronósticos en el mieloma múltiple. Además, sugieren un método que combina la evaluación de la clonalidad con inmunofenotipificación, tanto para el seguimiento del paciente con la enfermedad instaurada como para aquellos recién diagnosticados, cuyo panel incluye los antígenos Ig $\lambda$ citoplasmática/Igk citoplasmática/CD19/CD56/CD38/CD45 y los antígenos Ig $\lambda$ citoplasmática/Igk citoplasmática/CD19/CD138/CD38/CD45 [59].
Entre los hallazgos importantes se encuentra que el marcador CD10 tiene una expresión baja y el CD9 una expresión alta en la gammapatía monoclonal de significado incierto [60]. El CD22 es indetectable, tanto en las células plasmáticas normales como en las neoplásicas; el CD20 es de expresión negativa en las células plasmáticas normales, pero se ha observado en algunos subgrupos de mieloma múltiple asociado a morfología de células plasmáticas maduras y $t(11 ; 14)[61,62]$. La expresión del CD19 en las células plasmáticas de la gammapatía 
monoclonal de significado incierto se observa disminuida en comparación con la de las células plasmáticas normales, y aumentada respecto a las células plasmáticas del mieloma múltiple; así mismo, la pérdida de su expresión se asocia con la progresión de la enfermedad [63].

La expresión de la molécula CD27 es similar en la gammapatía monoclonal de significado incierto y en las células plasmáticas normales, pero la pérdida de su expresión es un factor propio de las células del mieloma múltiple en estadios II y III, lo que indica un valor pronóstico de este marcador [59]. El CD28 es de expresión normal en las células linfoides T y no se expresa en las células plasmáticas normales; sin embargo, se expresa brillante en las células plasmáticas neoplásicas del mieloma múltiple, encontrándose tanto en los pacientes con enfermedad avanzada como en aquellos en recaída. Por su parte, este marcador tiene baja expresión en la gammapatía monoclonal de significado incierto, lo que es de utilidad para establecer el diagnóstico diferencial [64].

Cuando las células plasmáticas son malignas pueden adquirir y expresar marcadores no propios tales como las moléculas CD28, CD33, CD56 y CD117, y perder la expresión de la molécula CD27 [65]. La expresión del marcador mieloide CD33 se ha encontrado hasta en un $18 \%$ de los pacientes con mieloma múltiple y se ha relacionado con la presencia de plasmoblastos anómalos y células plasmáticas inmaduras, pobremente diferenciadas y con gran capacidad infiltrante, así como con el aumento en los niveles de la lactacto deshidrogenasa y la $\beta 2$-microglobulina sérica. Por su parte, la expresión de la molécula CD13 se ha evidenciado hasta en el $53 \%$ de las células plasmáticas neoplásicas con patrón focal y difuso de infiltración. Ambos marcadores mieloides son indicativos de mal pronóstico [66]. El CD117 es un marcador que no se observa en las células plasmáticas normales y que se ha encontrado con mayor expresión en la gammapatía monoclonal de significado incierto, respecto al mieloma múltiple, por lo que su expresión se considera de mejor pronóstico para el paciente [59].

Las células plasmáticas exhiben una restricción isotípica, es decir, que cada una de ellas solo puede producir un único tipo de inmunoglobulina, mientras que las células plasmáticas clonales malignas pueden expresar, además, de forma homogénea, cadenas ligeras kappa o lambda de las inmunoglobulinas, que pueden ser identificadas en muestras de plasma u orina con un patrón electroforético de pico anormal. Además, en médula ósea estas células clonales pueden mostrar diversos inmunofenotipos [65].

En la mayoría de los casos, las células plasmáticas del mieloma múltiple expresan un fenotipo inmunoglobulina citoplasmática + , $\mathrm{CD} 38+, \mathrm{CD} 45-, \mathrm{CD} 138+, \mathrm{CD} 56+$ y CD19 +; solo una pequeña proporción expresan CD10 y HLA-DR, y el 20\% CD20 [67]. En algunas células clonales la expresión del CD38 se puede observar disminuida en comparación con las células plasmáticas normales. La presencia del CD56 se correlaciona con la agresividad de la enfermedad, ya que acelera el proceso de metástasis[59].

La clonalidad de las células plasmáticas se establece utilizando el cociente $\mathrm{k} / \lambda$, cuyo valor normal es de 0,26 a 1,65. En el mieloma múltiple y las gammapatías monoclonales esta relación es anormal, donde una relación menor a 1:2, con un promedio menor de 0,26 , es indicativa de una población clonal con cadenas livianas $\lambda$, mientras que 
si la relación es mayor de $4: 1$, con un promedio mayor de 1,65, indica la presencia de una población clonal con cadenas livianas $\mathrm{K}$. Esto es útil para diferenciar la proliferación monoclonal de células plasmáticas del mieloma múltiple de la plasmocitosis reactiva, relacionada con enfermedades del tejido conjuntivo, carcinoma metastásico, hepatopatía e infección [68].

Para realizar el diagnóstico diferencial entre el mieloma múltiple y las gammapatías monoclonales de significado incierto se considera la cantidad de células plasmáticas dentro del porcentaje total de leucocitos, el inmunofenotipo, la clonalidad y la cantidad anormal de células plasmáticas del total de células plasmáticas evaluadas por citometría de flujo. Como marcadores pronósticos se considera la expresión específica de CD45/CD56/CD117 y CD28. Para la detección de la enfermedad mínima residual, en pacientes con mieloma múltiple, se pesquisa la expresión anormal de las cadenas $\mathrm{k} / \lambda$ de la inmunoglobulina y el porcentaje de células plasmáticas totales dentro del recuento del total de leucocitos [67].

\section{Diagnóstico y pronóstico del mieloma múltiple}

El mieloma múltiple se caracteriza por plasmocitosis de la médula ósea, con agregados de células plasmáticas, que presentan atipia citológica y constituyen más del $30 \%$ de la celularidad total. Rara vez, los pacientes con mieloma múltiple muestran un marcado aumento en las células plasmáticas circulantes en sangre periférica [69].

La estadificación de los pacientes con mieloma múltiple se ha realizado tradicionalmente según la clasificación propuesta, en 1975, por Durie y Salmon [70], la cual incluye los siguientes criterios: nivel de hemoglobina, nivel de calcio sérico, radiología ósea y niveles de producción de proteína monoclonal. Esta clasificación permite establecer tres estadios, donde el estadio I cursa con baja masa tumoral, el II con masa tumoral intermedia y el III con alta masa tumoral. A su vez, cada estadio se subclasifica en $A$ o $B$ según la función renal, y en grados de afectación ósea que van desde el 0 al 3. El Índice Pronóstico Internacional (IPS) es un sistema de clasificación propuesto recientemente, y con gran aceptación, el cual establece una serie de estadios que brindan información de la supervivencia estimada de los pacientes y los divide en tres grupos de riesgo de acuerdo con los niveles de $\beta 2$-microglobulina y de albúmina, donde el grupo I es de riesgo bajo, el II es de riesgo intermedio y el III es de riesgo alto $[69,71,72]$.

Respecto a los factores pronósticos están aquellos relacionados con la carga tumoral, entre los que se incluyen los niveles de la $\beta 2$-microglobulina, la lactato deshidrogenasa y el estadio del índice pronóstico internacional, y los intrínsecos al tumor, como son la morfología de las células plasmáticas, la actividad proliferativa de las células plasmáticas y las características citogenéticas que clasifican a los pacientes en tres grupos: bajo, intermedio y alto riesgo [73].

La clasificación del mieloma múltiple, de acuerdo con su perfil de riesgo genético, lo considera de alto riesgo ante la existencia de cualquiera de las siguientes alteraciones genéticas: $t(4 ; 14), t(14 ; 16), t(14 ; 20)$, deleción de $17 p$ (determinado mediante hibridación fluorescente in situ - FISH), deleción de $13 q$, hipodiploidías y amplificación del brazo largo del cromosoma $1 q+[74,75]$. 
El diagnóstico del mieloma múltiple requiere de la presencia de uno o más de los eventos que lo definen, como la evidencia de daño orgánico final debido a hipercalcemia, con calcio sérico mayor a $0,25 \mathrm{mmol} / \mathrm{L}$ (mayor que $1 \mathrm{mg} / \mathrm{dL}$ ) por encima del límite superior normal o mayor a $2,75 \mathrm{mmol} / \mathrm{L}$ (mayor que $11 \mathrm{mg} / \mathrm{dL}$ ), insuficiencia renal con aclaramiento de creatinina menor a $40 \mathrm{~mL} /$ min o creatinina sérica mayor a $177 \mu \mathrm{mol} / \mathrm{L}$ (mayor que $2 \mathrm{mg} / \mathrm{dL}$ ) y anemia con valor de hemoglobina mayor a $20 \mathrm{~g} / \mathrm{L}$, por debajo del límite inferior normal o con un valor menor a $100 \mathrm{~g} / \mathrm{L}$; además, evidencia del 10\% o más de células plasmáticas clonales en el examen de médula ósea o un plasmocitoma probado por biopsia [76]. Cabe mencionar que, como toda discrasia de células plasmáticas, su diagnóstico y clasificación depende de la correlación de los datos de las imágenes radiológicas, como las lesiones óseas, con los obtenidos por el laboratorio, como el tipo y cantidad de proteína $M$, y los hallazgos morfológicos, citogenéticos, fenotípicos y moleculares [77].

En el mieloma múltiple es necesaria la valoración del clínico acerca de si existe o no un daño de órgano terminal para establecer el diagnóstico de malignidad. De esta manera se considera que, los pacientes con una carga tumoral sustancial, sin llegar a ser muy elevada, tienen una "premalignidad", la cual puede corresponder a alguno de los dos estadios que le anteceden al mieloma, la gammapatía monoclonal de significado incierto o el mieloma múltiple indolente (SMM, del inglés, smoldering myeloma), en los que el paciente no recibe terapia hasta que se presenten pruebas de complicaciones graves relacionadas con la enfermedad sintomática. En el mieloma múltiple indolente los pacientes tienen un mayor riesgo de progresión a malignidad (aproximada- mente del $10 \%$ por año) respecto a la gammapatía monoclonal de significado incierto (aproximadamente del 1\% por año) $[16,78]$.

Partiendo de que hace algunos años se asumía que el requisito de daño de órgano terminal era único para definir una neoplasia maligna, que la mayoría de los pacientes con gammapatía monoclonal de significado incierto y mieloma múltiple indolente pueden encontrarse asintomáticos y sin progresión durante años en ausencia de terapia, y que no era posible realizar un tratamiento oportuno para minimizar o prevenir el daño orgánico, en el 2014 el Grupo Internacional de Trabajo sobre Mieloma (IMWG, por sus siglas en inglés) revisó la definición de mieloma múltiple de manera que fuera posible realizar un diagnóstico precoz, antes del daño del órgano terminal, y establecer el riesgo de progresión de la enfermedad.

Así como nuevos criterios diagnósticos del mieloma múltiple se incluyeron tres biomarcadores específicos: la plasmocitosis clonal de la médula ósea mayor o igual al $60 \%$, la proporción de cadenas ligeras libres en suero ( $r C L L$ ) mayor o igual a 100 (con una concentración de rCLL mayor o igual a 100 $\mathrm{mg} / \mathrm{L}$ ) y más de una lesión focal detectada por resonancia magnética. Según estudios llevados a cabo por Rajkumar y colaboradores (2014 y 2016), cada uno de estos nuevos biomarcadores está asociado con un riesgo de aproximadamente el $80 \%$ de progresión a daño de órgano terminal $[72,79]$.

\section{Conclusiones}

Es importante efectuar la valoración y descripción morfológica de las células clonales en el diagnóstico temprano de las neoplasias de células plasmáticas, específicamente del mieloma múltiple. En especial, la des- 
cripción de las alteraciones nucleares y la realización del recuento diferencial en el extendido del aspirado de médula ósea, donde se observe la presencia de células inmaduras, permitirán, potencialmente, intervenir en la decisión terapéutica y en el pronóstico del paciente. Por tal razón, se requiere documentar estos hallazgos y fortalecer el reporte de los plasmoblastos al momento de evaluar el aspirado de médula ósea.

El inmunofenotipo por citometría de flujo posibilita el diagnóstico diferencial entre las células plasmáticas normales o reactivas y las neoplásicas, así como la identificación de la expresión de anticuerpos aberrantes relacionados con el pronóstico de la enfermedad. Incluso, en pacientes que no presentan síntomas CRAB, permite obtener un diagnóstico oportuno, lo que se traduce en la disminución de los daños orgánicos, la aceleración del tratamiento y una probable mejoría en el pronóstico del paciente. Igualmente, la citometría de flujo es fundamental en el seguimiento del paciente en la búsqueda de enfermedad mínima residual.

\section{Bibliografía}

1. Gorczyca W. Plasma cell neoplasms. Atlas of Differential Diagnosis in Neoplastic Hematopathology (ed 3a). Boca Ratón, Florida, Estados Unidos: CRC Press; 2014: 359382.

2. Swerdlow SH, Campo E, Harris NL, Jaffe ES, Pileri SA, Stein $\mathbf{H}$, et al. WHO Classification of Tumours of Haematopoietic and Lymphoid Tissues (ed 4a). Lyon, Francia: International Agency for Research on Cancer; 2008.

3. San Miguel JF, Gutierrez NC, Mateo G, Orfao A. Conventional diagnostics in multiple myeloma. Eur J Cancer 2006; 42: 1510-1519.

4. Swerdlow SH, Campo E, Pileri SA, Harris NL, Stein H, Siebert R, et al. The 2016 revision of the World Health Organization classification of lymphoid neoplasms. Blood 2016; 127: 2375-2390.

5. Ramasamy $\mathbf{K}$, Lonial S. Fast Facts: Multiple Myeloma and Plasma Cell Dyscrasias. Oxford, Reino Unido: Health Press Limited; 2015.

6. Rajkumar SV. Multiple myeloma: 2016 update on diagnosis, risk-stratification, and management. Am J Hematol 2016; 91: 719-734.
7. Erikci AA, Ozturk A, Tekgunduz E, Sayan O. Acute myeloid leukemia complicating multiple myeloma: a case successfully treated with etoposide, thioguanine, and cytarabine. Clin Lymphoma Myeloma 2009; 9: E14-15.

8. Luca DC, Almanaseer IY. Simultaneous presentation of multiple myeloma and acute monocytic leukemia. Arch Pathol Lab Med 2003; 127: 1506-1508.

9. Mailankody S, Pfeiffer RM, Kristinsson SY, Korde N, Bjorkholm M, Goldin LR, et al. Risk of acute myeloid leukemia and myelodysplastic syndromes after multiple myeloma and its precursor disease (MGUS). Blood 2011; 118: 4086-4092.

10. Dincol G, Nalcaci M, Dogan O, Aktan M, Kucukkaya R, Agan M, et al. Coexistence of chronic neutrophilic leukemia with multiple myeloma. Leuk Lymphoma 2002; 43: 649-651.

11. Cid Ruzafa J, Merinopoulou E, Baggaley RF, Leighton $\mathbf{P}$ Werther W, Felici D, et al. Patient population with multiple myeloma and transitions across different lines of therapy in the USA: an epidemiologic model. Pharmacoepidemiol Drug Saf 2016; 25: 871-879.

12. Jemal A, Murray T, Samuels A, Ghafoor A, Ward E, Thun MJ. Cancer statistics, 2003. CA Cancer J Clin 2003; 53: 5-26.

13. Blade J, Kyle RA, Greipp PR. Multiple myeloma in patients younger than 30 years. Report of 10 cases and review of the literature. Arch Intern Med 1996; 156: 14631468.

14. El Mangad F EZ, El Bouchti I. Multiple Myeloma in Unusually Young Patient: A Case Report. Int J Clin Med 2014; 05: 890-893.

15. Ramón Rodríguez LG, Agramonte Llanes O, Hernández Padrón C, Espinosa Martínez E, Losada Buchillón R, Ávila Cabrera O, et al. Mieloma múltiple y embarazo: Primer reporte de caso en Cuba. Rev Cubana Hematol Inmunol Hemoter 2010; 26: 70-75.

16. Kyle RA, Gertz MA, Witzig TE, Lust JA, Lacy MQ, Dispenzieri A, et al. Review of 1027 patients with newly diagnosed multiple myeloma. Mayo Clin Proc 2003; 78: 21-33.

17. Rosenberg PS, Barker KA, Anderson WF. Future distribution of multiple myeloma in the United States by sex age, and race/ethnicity. Blood 2015; 125: 410-412.

18. Sant M, Allemani C, Tereanu C, De Angelis R, Capocaccia $\mathbf{R}$, Visser $\mathbf{O}$, et al. Incidence of hematologic malignancies in Europe by morphologic subtype: results of the HAEMACARE project. Blood 2010; 116: 3724-3734.

19. Siegel RL, Miller KD, Jemal A. Cancer statistics, 2016. CA Cancer J Clin 2016; 66: 7-30.

20. Teras LR, DeSantis CE, Cerhan JR, Morton LM, Jemal A, Flowers CR. 2016 US lymphoid malignancy statistics by World Health Organization subtypes. CA Cancer J Clin 2016.

21. Alvarado M, Álvarez JL, Anaya I, et al. Primer Consenso Nacional de Mieloma Múltiple por Hematólogos del ISSSTE. Rev Hematol México 2015; 16: 306-332.

22. World Health Organization, International Agency for Research on Cancer. Globocan 2012: estimated cancer incidence, mortality and prevalence worldwide in 2012 - Population Fact Sheets: Colombia. 2012. Disponible: http://globocan.iarc.fr/Pages/fact_sheets_population. aspx. 
23. World Health Organization, International Agency for Research on Cancer. Globocan 2012: estimated cancer incidence, mortality and prevalence worldwide in 2012 - Online Analysis: Prediction. 2012. Disponible: http:// globocan.iarc.fr/Pages/burden_sel.aspx.

24. Dispenzieri A. Myeloma: management of the newly diagnosed high-risk patient. Hematology Am Soc Hematol Educ Program 2016; 2016: 485-494.

25. Heusschen R, Muller J, Duray E, Withofs N, Bolomsky A, Baron F, et al. Molecular mechanisms, current management and next generation therapy in myeloma bone disease. Leuk Lymphoma 2018; 59: 14-28.

26. Roodman GD. Myeloma bone disease: pathogenesis and treatment. Oncology (Williston Park) 2005; 19: 983-984, 986.

27. Shah V, Sherborne AL, Walker BA, Johnson DC, Boyle EM, Ellis S, et al. Prediction of outcome in newly diagnosed myeloma: a meta-analysis of the molecular profiles of 1905 trial patients. Leukemia 2017; 32: 102.

28. Fonseca R, Abouzaid S, Bonafede M, Cai Q, Parikh K, Cosler L, et al. Trends in overall survival and costs of multiple myeloma, 2000-2014. Leukemia 2016; 31: 1915.

29. Moeremans K, Annemans L. An update: health economics of managing multiple myeloma. Eur J Cancer 2006; 42: 1684-1691.

30. Shaffer AL, Shapiro-Shelef $M$, Iwakoshi NN, Lee $A H$ Qian SB, Zhao H, et al. XBP1, downstream of Blimp-1, expands the secretory apparatus and other organelles, and increases protein synthesis in plasma cell differentiation. Immunity 2004; 21: 81-93.

31. Zandi S, Mansson R, Tsapogas P, Zetterblad J, Bryder D, Sigvardsson M. EBF1 is essential for B-lineage priming and establishment of a transcription factor network in common lymphoid progenitors. J Immunol 2008; 181: 3364-3372.

32. Shapiro-Shelef $\mathbf{M}$, Calame $\mathbf{K}$. Regulation of plasma-cell development. Nat Rev Immunol 2005; 5: 230-242.

33. Greer JP, Foerster J, Lukens JN, Rodgers GM, Paraskevas F, Glader B. Wintrobe's Clinical Hematology (ed 11a). Maryland, Estados Unidos: Lippincott Williams \& Wilkins; 2004.

34. Goldman L, Schafer Al. Goldman-Cecil. Tratado de Medicina Interna. Vol. 1 (ed 25a). España: Elsevier Inc.; 2017.

35. Porwit A, McCullough J, Erber W. Blood and bone marrow pathology: Churchill Livingstone Elsevier; 2011

36. Miale JB. Hematología : Medicina de Laboratorio (ed 6a). España: Reverté; 1985.

37. College of American Pathologists. 2017 Hematology, Clinical Microscopy, and Body Fluids Glossary. 2017. Disponible: http://www.cap.org/ShowProperty?nodePath=/UCMCon/Contribution\%20Folders/WebContent/ pdf/hematology-glossary.pdf.

38. Nikesitch $\mathbf{N}$, Ling SC. Molecular mechanisms in multiple myeloma drug resistance. J Clin Pathol 2016; 69: 97-101.

39. Morgan GJ, Walker BA, Davies FE. The genetic architecture of multiple myeloma. Nat Rev Cancer 2012; 12: 335348.

40. Yun Z, Zhichao J, Hao Y, Ou J, Ran Y, Wen D, et al. Targeting autophagy in multiple myeloma. Leuk Res 2017; 59: 97-104.

41. Ribourtout B, Zandecki M. Plasma cell morphology in multiple myeloma and related disorders. Morphologie 2015; 99: 38-62.

42. Ho WK, Zantomio D. Auer rod-like inclusions in plasma cells in multiple myeloma. J Clin Pathol 2014; 67: 547548.

43. Tejwani N, Tyagi S, Dass J. Multiple Auer Rod Like Inclusions in Multiple Myeloma. Indian J Hematol Blood Transfus 2017; 33: 121-122.

44. Hutter G, Nowak D, Blau IW, Thiel E. Auer rod-like intracytoplasmic inclusions in multiple myeloma. A case report and review of the literature. Int J Lab Hematol 2009; 31: $236-240$.

45. Zhu L, An L, Zhang XY, Ren XR, Song JW. Peroxidase-positive Auer bodies in plasma cells in multiple myeloma: a case report. Int J Clin Exp Pathol 2015; 8: 15301-15306.

46. Eyre TA, Littlewood TJ, Bain BJ. Dutcher bodies: cytoplasmic inclusions within the nucleus. Br J Haematol 2014; 166: $946-947$.

47. Ortíz-Hidalgo $\mathbf{C}$. De las células plasmáticas al mieloma múltiple. Una breve perspectiva histórica. Patol Rev Latinoam 2011; 49: 120-131.

48. Pantanowitz L, Tranovich V, Ballesteros E. Flaming plasma cells. Arch Pathol Lab Med 2001; 125: 1394-1395.

49. Seili-Bekafigo I, Valkovic T, Babarovic E, Duletic-Nacinovic A, Jonjic N. Myeloma cell morphology and morphometry in correlation with clinical stages and survival. Diagn Cytopathol 2013; 41: 947-954.

50. You E, Cho SY, Yang JJ, Lee HJ, Lee WI, Yoon HJ, et al. Plasma cell neoplasms showing multilobulated nuclei. Acta Haematol 2013; 130: 98-100.

51. Ghevaert C, Fournier M, Bernardi F, Genevieve F, Pouyol F, Zandecki M. Non-secretory multiple myeloma with multinucleated giant plasma cells. Leuk Lymphoma 1997; 27: 185-189.

52. Subramanian R, Basu D, Dutta TK. Prognostic significance of bone marrow histology in multiple myeloma. Indian J Cancer 2009; 46: 40-45.

53. Bartl R, Frisch B, Fateh-Moghadam A, Kettner G, Jaeger $\mathbf{K}$, Sommerfeld W. Histologic classification and staging of multiple myeloma. A retrospective and prospective study of 674 cases. Am J Clin Pathol 1987; 87: 342-355.

54. Paule B, Quillard J, Bisson M, Kahn MF, Massias P. Prognostic significance of plasma cell morphology in multiple myeloma. Nouv Rev Fr Hematol 1988; 30: 209-212.

55. Bartl R, Frisch B, Burkhardt R, Fateh-Moghadam A, Mahl $\mathbf{G}$, Gierster $\mathbf{P}$, et al. Bone marrow histology in myeloma: its importance in diagnosis, prognosis, classification and staging. Br J Haematol 1982; 51: 361-375.

56. Greipp PR, Raymond NM, Kyle RA, O'Fallon WM. Multiple myeloma: significance of plasmablastic subtype in morphological classification. Blood 1985; 65: 305-310.

57. De los Reyes N, Monserrat J, Martínez MV, Álvarez MR, Minguela A. Utilidad de la Citometría de Flujo en el diagnóstico y pronóstico del Mieloma Múltiple. Cuadernos de Hematología 2010: 1-12

58. Flores-Montero J, de Tute R, Paiva B, Perez JJ, Bottcher $\mathrm{S}$, Wind $\mathbf{H}$, et al. Immunophenotype of normal vs. myeloma plasma cells: Toward antibody panel specifications for MRD detection in multiple myeloma. Cytometry B Clin Cytom 2016; 90: 61-72.

59. Karthick R M, Kovarova L, Hajek R. Review of phenotypic 
markers used in flow cytometric analysis of MGUS and $\mathrm{MM}$, and applicability of flow cytometry in other plasma cell disorders. Br J Haematol 2010; 149: 334-351.

60. Zandecki M, Facon T, Bernardi F, Izydorczyk V, Dupond L, Francois M, et al. CD19 and immunophenotype of bone marrow plasma cells in monoclonal gammopathy of undetermined significance. J Clin Pathol 1995; 48: 548-552.

61. Lin P, Owens R, Tricot G, Wilson CS. Flow cytometric immunophenotypic analysis of 306 cases of multiple myeloma. Am J Clin Pathol 2004; 121: 482-488.

62. An G, Xu Y, Shi L, Zou D, Deng S, Sui W, et al. $t(11 ; 14)$ multiple myeloma: a subtype associated with distinct immunological features, immunophenotypic characteristics but divergent outcome. Leuk Res 2013; 37: 1251-1257.

63. Olteanu H, Wang HY, Chen W, McKenna RW, Karandikar NJ. Immunophenotypic studies of monoclonal gammopathy of undetermined significance. BMC Clin Pathol 2008; 8: 13.

64. Nair JR, Carlson LM, Koorella C, Rozanski CH, Byrne GE, Bergsagel PL, et al. CD28 expressed on malignant plasma cells induces a prosurvival and immunosuppressive microenvironment. J Immunol 2011; 187: 1243-1253.

65. Robillard $\mathbf{N}$, Wuillème $\mathrm{S}$, Moreau $\mathrm{P}$, Béné $\mathbf{M C}$. Immunophenotype of Normal and Myelomatous Plasma-Cell Subsets. Front Immunol 2014; 5: 137.

66. Shim H, Ha JH, Lee H, Sohn JY, Kim HJ, Eom HS, et al. Expression of myeloid antigen in neoplastic plasma cells is related to adverse prognosis in patients with multiple myeloma. Biomed Res Int 2014; 2014: 893243.

67. Rawstron AC, Orfao A, Beksac M, Bezdickova L, Brooimans RA, Bumbea $\mathrm{H}$, et al. Report of the European Myeloma Network on multiparametric flow cytometry in multiple myeloma and related disorders. Haematologica 2008; 93: 431-438.

68. Davids MS, Murali MR, Kuter DJ. Serum free light chain analysis. Am J Hematol 2010; 85: 787-790.

69. The International Myeloma Working Group, Kyle RA, Child J, Anderson K, Barlogie B, Bataille R, et al. Criteria for the classification monoclonal gammopathies, multiple myeloma and related disorders: a report of the Inter- national Myeloma Working Group. Br J Haematol 2003; 121: 749-757.

70. Durie BG, Salmon SE. A clinical staging system for multiple myeloma. Correlation of measured myeloma cell mass with presenting clinical features, response to treatment, and survival. Cancer 1975; 36: 842-854.

71. Conté L G, Figueroa M G, Lois V V, Cabrera C ME, León R A, García L H, et al. Valor pronóstico del nuevo sistema de etapificación internacional en mieloma múltiple: Comparación con el sistema de Durie-Salmon. Rev Méd Chile 2008; 136: 7-12

72. Rajkumar SV, Dimopoulos MA, Palumbo A, Blade J, Merlini G, Mateos MV, et al. International Myeloma Working Group updated criteria for the diagnosis of multiple myeloma. Lancet Oncol 2014; 15: e538-548.

73. Sociedad Argentina de Hematología. Guías de diagnóstico y tratamiento Clin Chiest Med 2015; 20: 335-337.

74. Sonneveld $\mathrm{P}$, Avet-Loiseau $\mathrm{H}$, Lonial $\mathrm{S}$, Usmani $\mathrm{S}$, Siegel D, Anderson KC, et al. Treatment of multiple myeloma with high-risk cytogenetics: a consensus of the International Myeloma Working Group. Blood 2016; 127: 29552962.

75. Kaufman GP, Gertz MA, Dispenzieri A, Lacy MQ, Buadi FK, Dingli D, et al. Impact of cytogenetic classification on outcomes following early high-dose therapy in multiple myeloma. Leukemia 2016; 30: 633-639.

76. Moreau P, San Miguel J, Sonneveld P, Mateos MV, Zamagni E, Avet-Loiseau $\mathrm{H}$, et al. Multiple myeloma: ESMO Clinical Practice Guidelines for diagnosis, treatment and follow-upt. Ann Oncol 2017; 28: iv52-iv61.

77. Cogbill $\mathbf{C H}$, Spears MD, Vantuinen $\mathrm{P}$, Harrington AM, Olteanu H, Kroft SH. Morphologic and cytogenetic variables affect the flow cytometric recovery of plasma cell myeloma cells in bone marrow aspirates. Int J Lab Hematol 2015; 37: 797-808.

78. Kyle RA, Rajkumar SV. Multiple myeloma. Blood 2008 111: 2962-2972.

79. Rajkumar SV. Updated Diagnostic Criteria and Staging System for Multiple Myeloma. Am Soc Clin Oncol Educ Book 2016; 35: e418-423.

\begin{abstract}
The advance in the knowledge of the pathophysiology of multiple myeloma has allowed the approach to the understanding of its heterogeneity in terms of clinical, cytomorphological, phenotypic, biochemical, genetic and molecular behavior, contributing to the development of new treatments and the establishment of models that point to a chronicity of the disease. The morphological changes of the plasma cell in the multiple myeloma indicate mainly to alterations at the nucleus level, which orient to the differentiation between reactive cells and clonal cells; also that their immaturity is of poor prognosis. Likewise, the expression of certain monoclonal markers, and the absence of others, detected by flow cytometry, immunophenotypically classify the neoplastic cells of multiple myeloma, which allows to considering this tool as one of the best in the diagnosis and management of minimal residual disease. In this review it is intend to emphasize the importance of identifying the morphological change that occur in plasma cells and their relationship with the immunophenotypics characteristics in neoplasia that have an important prognostic association with the multiple myeloma development.
\end{abstract}

Key words: Multiple Myeloma, immunophenotype, morphology, plasma cells, flow cytometry, bone marrow. 